\title{
Recomendações para amamentação no contexto do Covid-19: uma revisão integrativa
}

\author{
Breastfeeding recomendations in the Covid-19 context: an integrative review
}

Recomendaciones para una lactancia en el contexto del Covid-19: revision integral

Bruna Nunes Costa Lima Rosado ${ }^{1 *}$, Alda Maria da Silva², Ivana Cristina Vieira de Lima Maia ${ }^{3}$, Bruno Rafael Sousa Rosado ${ }^{4}$, Jéssica Karen de Oliveira Maia ${ }^{5}$, Emanuela Gomes Falcão ${ }^{3}$.

\section{RESUMO}

Objetivo: Analisar as recomendações sobre amamentação durante a pandemia de COVID-19. Métodos: Trata-se de revisão integrativa realizada nas seguintes bases de dados: MEDLINE/PubMed; Web of Science; LILACS, CINAHL, Cochrane Collaboration. Houve combinação dos seguintes descritores controlados: P (Postpartum Period, Período Pósparto); I (Breast Feeding, Amamentação); Co (Coronavirus Infections, Infecção por Coronavírus). Incluíram-se apenas artigos disponíveis na íntegra, independentemente do idioma e ano de publicação, que respondiam à questão norteadora. Resultados: Os 12 estudos selecionados foram publicados em 2020 e realizados principalmente nos Estados Unidos da América e Espanha. Quanto ao tipo de estudo, destacaram-se os estudos de revisão, seguido pelos estudos transversais e a abordagem quantitativa. Os estudos apresentam informações controversas sobre a suspensão ou manutenção da amamentação em casos de mãe positiva para COVID-19, orientando a decisão individualizada por parte de pais e profissionais, levando em consideração o consentimento dos pais, o estado de saúde da mãe e do recém-nascido, além da capacidade de cuidar do recém-nascido, resultados dos exames e a situação epidemiológica local. Considerações finais: As evidências indicaram que deve ser estimulada a amamentação de neonatos e bebês, porém as medidas de precauções devem ser mantidas e reforçadas criteriosamente.

Palavras-chaves: Período pós-parto, Amamentação, Infecção por coronavírus.

\section{ABSTRACT}

Objective: To analyze the recommendations on breastfeeding during the COVID-19 pandemic. Methods: This is an integrative review carried out in the following databases: MEDLINE / PubMed; Web Of Science; LILACS, CINAHL, Cochrane Collaboration. There was a combination of the following controlled descriptors: P (Postpartum Period, Postpartum Period); I (Breast Feeding); Co (Coronavirus Infections). Only articles available in full were included, regardless of language and year of publication, which answered the guiding question. Results: The 12 selected studies were published in 2020 and carried out mainly in the United States of America and Spain. As for the type of study, review studies stood out, followed by cross-sectional studies and the quantitative approach. The studies present controversial information on the suspension or maintenance of breastfeeding in cases of a positive mother for COVID-19, guiding the individualized decision by parents and professionals, taking into account the consent of the parents, the health status of the mother and the newborn-birth, in addition to the ability to care for the newborn, test results and the local epidemiological situation. Final considerations: The evidence indicated that breastfeeding for newborns and babies should be encouraged, but precautionary measures should be carefully maintained and reinforced.

Keywords: Postpartum period, Breast feeding, Coronavirus infections.

\section{RESUMEN}

Objetivo: Analizar las recomendaciones sobre lactancia materna durante la pandemia de COVID-19. Métodos: Una revisión integradora realizada en las siguientes bases de datos: MEDLINE / PubMed; Web Of Science (WOS); LILACS, CINAHL, Cochrane Collaboration. Hubo una combinación de los siguientes descriptores controlados: P (período posparto, período posparto); Yo (lactancia materna); Co (Infecciones por coronavirus). Solo se incluyeron los artículos disponibles en su totalidad, independientemente del idioma y año de publicación, que respondieron a la pregunta orientadora. Resultados: Los 12 estudios seleccionados fueron publicados en 2020 y realizados principalmente en Estados Unidos de América y España. En cuanto al tipo de estudio, se destacaron los estudios de revisión, seguidos de los estudios transversales y el abordaje cuantitativo. Los estudios presentan información controvertida sobre la suspensión o mantenimiento de la lactancia materna en casos de madre positiva para COVID-19, orientando la decisión individualizada de padres y profesionales, teniendo en cuenta el consentimiento de los padres, el estado de salud de la madre y el recién nacido. -nacimiento, además de la capacidad de cuidar al recién nacido, los resultados de las pruebas y la situación epidemiológica local. Consideraciones finales: La evidencia indicó que se debe fomentar la lactancia materna en recién nacidos y bebés, pero se deben mantener y reforzar cuidadosamente las medidas de precaución.

Palabras clave: Puerperio, Lactancia materna, Infección por coronavirus.

${ }^{1}$ Faculdade IDE, Recife - PE. *E-mail: bruninhahnunes@hotmail.com

2 Escola de Saúde Pública, Fortaleza - CE.

3 Universidade Estadual do Ceará (UECE), Fortaleza - CE.

${ }^{4}$ Hospital da Restauração, Secretaria de Saúde do Estado de Pernambuco (HR/SES-PE), Recife - PE.

5 Universidade Federal do Ceará (UFC), Fortaleza - CE. 


\section{INTRODUÇÃO}

No final de dezembro do ano de 2019, foram diagnosticados vários casos de pneumonia de origem desconhecida em Wuhan, na China. Em janeiro de 2020 foi descoberto que se tratava de uma nova linhagem de Coronavírus, responsável pela Síndrome Respiratória Aguda Grave (do inglês Severe Acute Respiratory Syndrome - SARS), sendo a doença nomeada SARS-COV-2, ou Coronavirus Disease 2019 (COVID-19). A doença conseguiu ultrapassar os limites territoriais chineses, causando uma devastadora pandemia, com alto índice de mortalidade que varia de acordo com as características epidemiológicas e sociais de cada país (LIU X, et al., 2020; MASCARENHAS VHA, et al., 2020).

Sua rápida proliferação levou a Organização Mundial de Saúde (OMS) a declarar, em 30 de janeiro de 2020, o estado de "Emergência Pública de Importância Internacional". Até o final do mês de junho, foram diagnosticados mais de 10 milhões de casos e 500.000 mil mortes no mundo. Sem dúvidas a COVID-19 elevou a um nível muito alto o risco global e tornou-se um problema de saúde pública (LIU X, et al., 2020; MASCARENHAS VHA, et al., 2020).

A transmissão ocorre por meio de gotículas das vias respiratórias de pessoas sintomáticas ou assintomáticas portadores do vírus, e por objetos contaminados por eles. Há também evidências de contaminação pelas fezes. No intuito de evitar que a maioria de população seja contaminada ao mesmo tempo e o sistema de saúde seja sobrecarregado, a OMS e o Ministério da Saúde têm recomendado o isolamento social, detecção precoce da infecção, notificação, investigação e manejo adequado dos casos (MASCARENHAS VHA, et al., 2020).

A maioria dos infectados tem sintomas leves ou moderados e cerca de $15 \%$ podem desenvolver sintomas graves que requeiram suporte de oxigênio. Os pacientes que costumam apresentar maiores complicações são idosos, imunossuprimidos, obesos, gestantes, puérperas e pessoas com comorbidades (BRASIL, 2020; MASCARENHAS VHA, et al., 2020).

As gestantes e puérperas foram incluídas neste grupo por serem mais suscetíveis a complicações e resultados adversos. Isso foi observado em pandemias anteriores como a de Síndrome respiratória aguda grave (SARS) e Síndrome Respiratória do Oriente Médio (MERS). As mudanças fisiológicas e imunológicas que ocorrem neste período as deixam mais propensas a desenvolver infecções respiratórias. Ainda não há evidências que confirmem a transmissão vertical do SARs-CoV-2, embora já tenham sido observados alguns sinais de alterações placentárias decorrentes de processo inflamatório cuja suspeita recaem sobre a COVID19. O vírus não foi encontrado nas amostras de líquido amniótico, cordão umbilical, swab de orofaringe de neonatos e no leite materno (OMS, 2020; LIMA ACMACC, et al., 2020; YASSA M, et al., 2020).

O aleitamento materno constitui-se em uma forma natural de formação de vínculo, afeto, proteção e nutrição para criança, sendo reconhecida como a intervenção mais sensível, econômica e eficaz para redução da morbimortalidade infantil, além de trazer grandes benefícios para a mulher como a redução do risco de depressão pós-parto, anemia e outras patologias. Deve ser iniciado nas primeiras horas de vida do bebê, sendo o único alimento ofertado durante os seis primeiros meses e continuado até os 2 anos junto com a alimentação complementar (OMS, 2020; LIMA ACMACC, et al., 2020; GUIMARÃES DC, et al., 2019; MOCELIN HJS, et al., 2020)

O leite é um alimento natural, rico em gorduras, proteínas, imunológicos e demais nutrientes que trazem grandes benefícios para os lactentes. Por ser de grande importância durante os primeiros meses do nascimento, é recomendado que mulheres com suspeita ou diagnóstico confirmado de COVID-19 continuem a amamentação, levando em conta que os benefícios superam os riscos potenciais de transmissão a partir do leite materno (MARTINS ABM, et al., 2020; LIMA ACMACC, et al., 2020).

Evidências demonstram a importância de orientar a mulher sobre os benefícios dessa prática. Além disso, deve-se permitir que a mãe o recém-nascido $(R N)$ permaneçam em alojamento conjunto durante o período de internação e que o contato pele a pele seja estimulado, mesmo que elas ou o RN tenham suspeita ou confirmação de COVID-19 (BRASIL, 2020). 
De acordo as recomendações do Ministério da Saúde do Brasil, caso a mulher não se sinta segura em amamentar enquanto estiver contaminada com coronavírus, recomenda-se que ela seja orientada sobre a retirada do leite e a oferta ao seu filho por meio de copinho, xícara ou colher. Visto que a mãe infectada pode transmitir o vírus através de gotículas durante o contato com a criança, este órgão recomenda que algumas precauções sejam tomadas, dentre elas podemos citar: lavar as mãos antes de tocar no bebê ou retirar 0 leite, usar máscara facial durante a mamada e evitar falar ou tossir (BRASIL, 2020).

Os estudos disponíveis na literatura abordam de forma geral os cuidados que devem ser empregados com a mulher e o RN durante a amamentação, a fim de evitar a transmissão do COVID-19, mas são escassos os estudos que abordam as recomendações sobre os cuidados que devem ser adotados durante a prática do aleitamento materno (TACLA MT, et al., 2020).

Diante desse contexto, emergiu a seguinte questão norteadora: Quais são as recomendações sobre amamentação durante a pandemia de COVID-19? Como a infecção por COVID-19 é uma doença emergente, estudos dessa natureza são importantes para embasar a prática dos profissionais que atuam na área da saúde materno-infantil, tendo em vista que o aleitamento materno é uma prática com vários benefícios para o binômio mãe e filho, sendo importante que seja realizada de forma segura. A presente pesquisa tem como objetivo analisar as recomendações sobre amamentação durante a pandemia de COVID-19.

\section{MÉTODOS}

Trata-se de revisão integrativa, método que busca reunir e sintetizar resultados obtidos em pesquisas sobre um determinado tema ou questão, de maneira sistemática e ordenada, contribuindo para o aprofundamento do conhecimento do tema investigado. O estudo seguiu as seguintes etapas: identificação do tema e elaboração da questão de pesquisa, definição de critérios para inclusão e exclusão de estudos, determinação das informações a serem extraídas dos estudos selecionados, análise dos estudos incluídos, interpretação dos resultados e apresentação da síntese do conhecimento (MENDES KDS, et al., 2009).

A construção da questão de pesquisa baseou-se na estratégia PICo ( $P$ - participantes; I - fenômeno de interesse; Co - contexto do estudo), em que Participantes (P): mulheres no período pós-parto, (I): amamentação, (Co): Pandemia de COVID-19. A partir dessa estratégia, elaborou-se a seguinte questão de partida: Quais são as recomendações sobre amamentação durante a pandemia de COVID-19? (MIRANDA PIG, et al., 2021)

Incluíram-se apenas artigos disponíveis na íntegra, independentemente do idioma e ano de publicação, que respondiam à questão norteadora. Como se trata de um assunto recente, com escassez de publicações, também foram incluídos os estudos de revisão. Os critérios de exclusão aplicados consistiram em: artigos repetidos, indisponíveis, carta ao editor, boletins, anais de congresso e estudos que não respondessem diretamente à questão de pesquisa.

A busca dos artigos aconteceu no dia 12 de janeiro de 2021, sendo realizada por meio de buscadores de internet nas seguintes bases de dados: MEDLI-NE, via PubMed; WOS; LILACS, CINAHL, Cochrane Coleboration.

Inicialmente, a estratégia para busca dos estudos foi composta pela combinação dos seguintes descritores controlados cadastrados nos Descritores em Saúde (DeCS) e no Medical Subject Headings (MeSH Terms): P (Postpartum Period, Período Pós-parto); I (Breast Feeding, Amamentação); Co (Coronavirus Infections, Infecção por Coronavírus). Como estratégia de busca foram utilizados os descritores controlados por intermédio do operador booleano AND. Destaca-se que a busca foi realizada a partir dos descritores identificados e com sentido ampliado, sem uso de filtros, de modo a preservar amostras significativas e garantir menor risco de perdas.

Encontraram-se 72 artigos (Pubmed:8; Web of Science: 16; Cochrane:12; CINAHL: 36) os quais foram submetidos à leitura de títulos e resumos, com objetivo de refinar a busca. Destes, foram excluídos 5 artigos repetidos, 1 indisponível, 9 cartas ao editor, 1 boletim, 1 consenso, 1 editorial e 21 estudos que não respondessem diretamente à questão de pesquisa. Mantiveram-se 33 artigos (Pubmed: 3; Web of Sciense: 
9; Cochrane: 0; CINAHL: 21) que foram lidos na íntegra, sendo a amostra final composta por 11 artigos. 0 processo de seleção dos estudos foi realizado por duas pesquisadoras, de forma independente e, em caso de divergência, houve debate para estabelecimento de consenso. Foi elaborado um fluxograma relativo à busca dos artigos nas bases de dados (Figura 1).

Figura 1 - Fluxograma de descrição dos artigos encontrados, excluídos e selecionados de acordo com cada base de dados.

\begin{tabular}{|c|c|c|}
\hline 喆 & $\begin{array}{l}\text { Estudos identificados nas bases } \\
\text { de dados: } \\
\text { Medline/Pubmed: } 08 \\
\text { Web of Science: } 16 \\
\text { Cochrane: } 12 \\
\text { CINAHL: } 36 \\
\text { Total: } 72\end{array}$ & $\begin{array}{l}\text { Exclusão de estudos: } \\
\text { - Medline/Pubmed: } 05 \text { ( } 03 \text { cartas para } \circ \text { Editor, } 01 \text { não disponivel } \\
\text { na integra, } 01 \text { editorial). } \\
\text { - Web of Science: } 07 \text { ( } 05 \text { artigos não contemplam o tema em estudo, } \\
01 \text { consenso, } 01 \text { boletim médico). }\end{array}$ \\
\hline 密 & $\begin{array}{l}\text { Artigos para avaliação de } \\
\text { elegibilidade }(n=33)\end{array}$ & $\begin{array}{l}\text { - Cochrane: } 12 \text { (não respondiam à questão norteadora). } \\
\text { - CINAHL: } 15 \text { ( } 5 \text { repetidos, } 6 \text { cartas ao editor, não respondem } \\
\text { diretamente à questão de pesquisa) }\end{array}$ \\
\hline
\end{tabular}
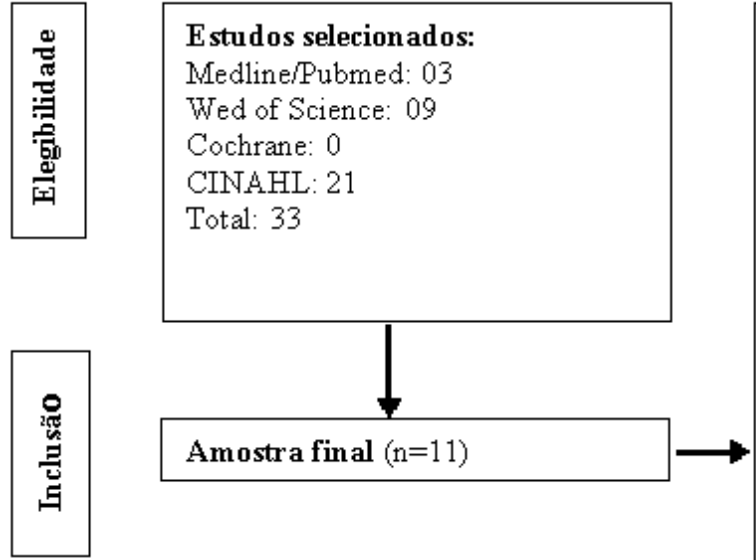

Exclusão de estudos:

- Medline/Pubmed: 01(Aborda a experiência do parto e pós-parto durante o periodo de confinamento)

- Web Of Science: 02 abordam a temática de complicações maternas e neonatais decorrentes do COVID -19

- CINAHL: 01 (aborda a pasteurização em tempos de COVID-19); 01 (enfatiza as complicaçóes maternas e fetais no contexto do COVID-19); 01 (infecção hospitalar e efeitos negativos associados a pandemia); 01 (medidas de prevenção utilizadas nas unidades neonatais); 14 (encontra-se entre os repetidos ou não respondem ao objetivo da pesquisa)

Fonte: Rosado BNCL, et al., 2021.

Os dados colhidos nos artigos selecionados foram analisados de forma descritiva, originando um quadro sinóptico de caracterização dos estudos (autores, ano, país de realização do estudo, objetivo, tipo de estudo, nível de evidência e principais resultados e conclusão). Para classificação do nível de evidência, utilizou-se a hierarquia de evidências proposta por Melnyk e Fineout-Overholt (Quadro 1). Esta hierarquia classifica os níveis I e II como fortes, III a V como moderados e VI a VII como fracos. (MELNYK; FINEOUT-OVERHOLT, 2011).

Quadro 1 - Hierarquia do nível de evidência.

\begin{tabular}{|c|c|}
\hline Classificação & Nível de Evidências \\
\hline I & Revisões sistemáticas e metanálises de ensaios clínicos randomizados. \\
\hline II & Ensaios clínicos randomizados. \\
\hline III & Ensaios controlados não randomizados. \\
\hline IV & Estudos caso-controle ou coorte. \\
\hline V & Revisões sistemáticas de estudos qualitativos ou descritivos. \\
\hline VI & Estudos qualitativos ou descritivos. \\
\hline VII & Parecer de autoridades e/ou relatórios de comitês de especialistas. \\
\hline
\end{tabular}

Fonte: Rosado BNCL, et al., 2021. 


\section{RESULTADOS}

Os 12 estudos selecionados foram publicados em 2020 e realizados nos seguintes países: Estados Unidos da América $(n=4)$, Espanha $(n=2)$, Itália $(n=1)$, África do Sul $(n=1)$, Irã $(n=1)$, Panamá $(n=1)$, e China $(n=1)$. Quanto ao tipo de estudo, destacaram-se os estudos de revisão $(n=7)$, seguido pelos estudos transversais $(n=4)$ e a abordagem quantitativa $(n=4)$ (Quadro 2).

Quadro 2 - Caracterização dos estudos quanto aos autores, ano, país, objetivo, tipo de estudo, nível de evidência, resultados e conclusões.

\begin{tabular}{|c|c|c|c|c|}
\hline Autores/ano & País & Objetivo & $\begin{array}{l}\text { Tipo de } \\
\text { estudo }\end{array}$ & $\begin{array}{c}\text { Nível de } \\
\text { evidência }\end{array}$ \\
\hline $\begin{array}{l}\text { Salvatore CM, } \\
\text { et al., } 2020\end{array}$ & $\begin{array}{l}\text { Estados } \\
\text { Unidos da } \\
\text { América }\end{array}$ & $\begin{array}{l}\text { Acompanhar neonatos nascidos de mães } \\
\text { positivas para SARS-CoV-2 no momento da } \\
\text { entrega, para elucidar melhores práticas em } \\
\text { relação ao controle de infecção e } \\
\text { identificação fatores de risco potenciais } \\
\text { associados à transmissão. }\end{array}$ & $\begin{array}{l}\text { Estudo de } \\
\text { coorte } \\
\text { observacional }\end{array}$ & IV \\
\hline $\begin{array}{c}\text { Lubbe W, et al., } \\
2020\end{array}$ & $\begin{array}{l}\text { África do } \\
\text { Sul }\end{array}$ & $\begin{array}{l}\text { Fornecer diretrizes para a gestão da } \\
\text { amamentação no domicílio e nas unidades } \\
\text { de saúde, incluindo nos cuidados intensivos } \\
\text { neonatais e de alto risco. }\end{array}$ & $\begin{array}{l}\text { Revisão de } \\
\text { literatura }\end{array}$ & VI \\
\hline $\begin{array}{l}\text { Cojocaru L, et } \\
\text { al., } 2020\end{array}$ & $\begin{array}{l}\text { Estados } \\
\text { Unidos da } \\
\text { América }\end{array}$ & $\begin{array}{l}\text { Avaliar a segurança do vínculo materno em } \\
\text { todos as pacientes positivas para SARS- } \\
\text { CoV-2. }\end{array}$ & $\begin{array}{l}\text { Estudo } \\
\text { Transversal }\end{array}$ & VI \\
\hline $\begin{array}{l}\text { Centeno- } \\
\text { Tablante E, et } \\
\text { al., } 2020\end{array}$ & $\begin{array}{l}\text { Estados } \\
\text { Unidos da } \\
\text { América }\end{array}$ & $\begin{array}{l}\text { Avaliar as evidências disponíveis } \\
\text { relacionadas à possível transmissão do } \\
\text { SARS-CoV-2 através do leite materno e da } \\
\text { amamentação. }\end{array}$ & $\begin{array}{l}\text { Revisão } \\
\text { Sistemática }\end{array}$ & $\mathrm{V}$ \\
\hline $\begin{array}{l}\text { Sighaldeh SS, } \\
\text { Kalan ME, } 2020\end{array}$ & Irã & $\begin{array}{l}\text { Discutir como cuidar de um recém-nascido } \\
\text { de uma mãe suspeita ou infectada com } \\
\text { COVID-19 usando as evidências existentes. }\end{array}$ & $\begin{array}{l}\text { Revisão } \\
\text { Sistemática }\end{array}$ & V \\
\hline $\begin{array}{l}\text { Fernández- } \\
\text { Carrasco FJ, et } \\
\text { al., } 2020\end{array}$ & Espanha & $\begin{array}{l}\text { Conhecer o plano de ação sobre } \\
\text { amamentação } \\
\text { diante de um recém-nascido com SARS- } \\
\text { CoV-2 }\end{array}$ & $\begin{array}{l}\text { Revisão de } \\
\text { literatura }\end{array}$ & VI \\
\hline $\begin{array}{l}\text { Martins-Filho } \\
\text { PR, et al., } 2020\end{array}$ & Panamá & $\begin{array}{l}\text { Avaliar as evidências atuais relacionadas à } \\
\text { presença de SARS-CoV-2 no leite materno } \\
\text { de mulheres grávidas com COVID-19. }\end{array}$ & $\begin{array}{l}\text { Revisão } \\
\text { Sistemática }\end{array}$ & V \\
\hline $\begin{array}{l}\text { Giuliani C, et } \\
\text { al., } 2020\end{array}$ & Itália & $\begin{array}{l}\text { Analisar as principais recomendações sobre } \\
\text { os cuidados que devem ser tomados durante } \\
\text { a amamentação de uma paciente com } \\
\text { CoVID-19. }\end{array}$ & $\begin{array}{l}\text { Revisão } \\
\text { Sistemática }\end{array}$ & V \\
\hline $\begin{array}{l}\text { Perrine CG, et } \\
\text { al., } 2020\end{array}$ & Espanha & $\begin{array}{l}\text { Descrever os tipos de lactação das mães } \\
\text { COVID-19, para identificar se foram } \\
\text { adicionadas dificuldades e para avaliar o } \\
\text { risco de infecção dos recém-nascidos. }\end{array}$ & $\begin{array}{c}\text { Estudo } \\
\text { Transversal }\end{array}$ & VI \\
\hline $\begin{array}{c}\text { Zhu C, et al., } \\
2020\end{array}$ & China & $\begin{array}{l}\text { Relatar as características clínicas da } \\
\text { pneumonia por COVID-19 em mulheres no } \\
\text { período de puerpério e evidências de } \\
\text { eliminação da SARS-CoV-2 em seu leite } \\
\text { materno. }\end{array}$ & $\begin{array}{l}\text { Estudo } \\
\text { Transversal }\end{array}$ & $\mathrm{VI}$ \\
\hline $\begin{array}{l}\text { Lackey KA, et } \\
\text { al., } 2020\end{array}$ & $\begin{array}{l}\text { Estados } \\
\text { Unidos }\end{array}$ & $\begin{array}{l}\text { Avaliar as evidências publicadas sobre a } \\
\text { presença deste e de outros coronavírus } \\
\text { humanos no leite humano }\end{array}$ & $\begin{array}{c}\text { Revisão } \\
\text { bibliográfica }\end{array}$ & VI \\
\hline
\end{tabular}

Fonte: Rosado BNCL, et al., 2021. 
A análise dos estudos permitiu identificar as categorias apresentadas no Quadro 2. De um modo geral, os estudos apresentam informações controversas sobre a suspensão ou manutenção da amamentação em casos de mãe positiva para COVID-19, orientando a decisão individualizada por parte de pais e profissionais, levando em consideração o consentimento dos pais, o estado de saúde da mãe e do recém-nascido, além da capacidade de cuidar do recém-nascido, resultados dos exames e a situação epidemiológica local (Quadro 3).

Quadro 3 - Quadro de categorização dos estudos.

\begin{tabular}{|c|c|}
\hline Categoria & Referência \\
\hline $\begin{array}{l}\text { A amamentação pode ser mantida no alojamento conjunto, } \\
\text { desde que sejam estabelecidas precauções de medidas } \\
\text { para controle de infecções. }\end{array}$ & $\begin{array}{c}\text { Salvatore CM, et al. (2020) } \\
\text { Lubbe W, et al. (2020) } \\
\text { Cojocaru L, et al. (2020) } \\
\text { Fernández-Carrasco FJ, et al. (2020) } \\
\text { Giuliani C, et al. (2020) } \\
\text { Perrine CG, et al. (2020) }\end{array}$ \\
\hline $\begin{array}{l}\text { Deve-se separar o bebê da mãe com infecção confirmada } \\
\text { (ou suspeita) por COVID-19 por pelo menos } 2 \text { semanas. }\end{array}$ & $\begin{array}{l}\text { Sighaldeh SS e Kalan ME, (2020) } \\
\text { Perrine CG, et al., (2020) } \\
\text { Zhu C, et al.,(2020) }\end{array}$ \\
\hline $\begin{array}{l}\text { A decisão de separar o binômio mãe-filho deve ser } \\
\text { individualizada. }\end{array}$ & Giuliani C,et al. (2020) \\
\hline $\begin{array}{l}\text { Nos graves, a mãe deve receber apoio para ordenha do leite } \\
\text { ou receber leite provindo de bancos de leite humano. }\end{array}$ & $\begin{array}{l}\text { Lubbe W, et al. (2020) } \\
\text { Fernández-Carrasco FJ, et al. (2020) }\end{array}$ \\
\hline $\begin{array}{l}\text { Não há evidências suficientes sobre a transmissão da } \\
\text { SARS-CoV-2 através do leite materno. }\end{array}$ & $\begin{array}{l}\text { Centeno-Tablante E, et al. (2020) } \\
\text { Martins-Filho PR, et al. (2020) } \\
\text { Lackey KA, et al., (2020) }\end{array}$ \\
\hline
\end{tabular}

Fonte: Rosado BNCL, et al., 2021.

\section{DISCUSSÃO}

As orientações encontradas na busca dos artigos foram tratadas de forma provisória, visto que podem ser alteradas conforme as novas evidências que irão surgindo. Além disso, observou-se que algumas orientações mudam de acordo com o local de publicação, alguns autores concordando sobre a continuidade do aleitamento materno e outros divergem sobre a continuidade da prática (GOES FGB, et al., 2021).

Contudo, os autores são unânimes ao ressaltar os benefícios da amamentação para redução da morbimortalidade de neonatos, bebês e crianças. Destaca-se seu efeito protetor no caso de doenças infecciosas devido à passagem direta de anticorpos da mãe para o concepto, bem como de fatores antiinfecciosos e transferência de imunidade de longa duração (LUBBE W, et al., 2020).

Em tempos de pandemia de COVID-19 é importante conhecer o potencial de imunidade passiva, ou seja, aquela que é passada da mãe para o concepto durante a gravidez e amamentação. No leite materno de mulheres positivas para COVID-19 foram identificados anticorpos IgA específicos, porém ainda existe uma lacuna sobre o tempo de duração destes anticorpos (CENTENO-TABLANTE E, et al., 2020).

Outro ponto controverso na literatura é a questão da transmissão vertical do vírus, apesar de que a maioria das pesquisas realizadas até o momento não demonstrarem evidências desse tipo de transmissão para o SARS-COV-2. Na China foi realizado um estudo para avaliar por meio do teste RT-PCR a presença do SARSCOV-2 em amostras do líquido amniótico, sangue do cordão umbilical, esfregaço de garganta neonatal e leite materno de seis pacientes com o intuito de analisar o potencial de transmissão vertical de COVID-19 e não foi vista a presença do vírus em nenhuma dessas amostras (CHEN H, et al., 2020).

Outros estudos confirmam tal afirmação e relatam que até o momento não existem evidências que apontem a transmissão do vírus através do leite materno de mulheres infectadas, sendo assim orientada a continuidade dessa prática, pois seus benefícios superam o risco da transmissão (MARCHIORI GRS, et al., 2020).

Na literatura ainda é possível encontrar muitos dados controversos sobre as orientações que devem ser fornecidas às mulheres com COVID-19 sobre o aleitamento materno, aumentando a insegurança dessas 
pacientes sobre qual conduta seguir. Na Turquia foi realizado um estudo que observou que grande parte das gestantes não tinham informações sobre a amamentação durante a pandemia ou achavam que a amamentação não era segura durante a pandemia. (YASSA M, et al., 2020).

É necessário que os profissionais junto com os veículos de comunicações atuem orientando essas pacientes sobre todos os cuidados que devem ser instituídos nesse período, com estímulo ao vínculo entre mãe e bebê. Uma pesquisa realizada nos Estados Unidos da América apoia o estímulo ao vínculo da mãe com o recém-nascido $(R N)$ através do alojamento conjunto, com contato pele a pele e incentivo à amamentação (COJOCARU L, et al., 2020; NG QJ, et al., 2020)

Estabelecimentos que apoiam adoção de medidas que estimulam o maior contato mãe e bebê tem maiores benefícios, pois ao separar o binômio é gerada uma cascata de efeitos prejudiciais, tais como: estresse materno e fetal, interrupção da fisiologia neonatal, além da ansiedade materna. Dentre os benefícios do manejo conjunto de mães e RNs está o início precoce da amamentação e sua continuidade, além do aumento da confiança materna e proteção contra o estresse relacionado à mudança do papel dos pais para algumas mães (COJOCARU L, et al., 2020).

Assim, o manejo conjunto do binômio deve ser mantido, a fim de facilitar a interação e o início precoce da amamentação. A decisão de separar ou não deve ser individualizada, levando em consideração o consentimento dos pais, bom estado de saúde da mulher e seu filho e a capacidade de realizar o cuidado, resultado do teste RT-PCR, o contexto e a situação epidemiológica local da atual pandemia. Portanto, é de extrema importância que os prestadores do cuidado ao binômio discutam os benefícios da amamentação com os pais, com o objetivo de individualizar qualquer escolha de alimentação (GIULIANI C, et al., 2020).

Todas as mulheres devem realizar o teste RT-PCR antes do parto e a partir do resultado e do estado geral da mulher devem ser traçados os planos de cuidado. Caso apresentem o resultado positivo e tenham condições, o aleitamento materno deve ser estimulado. Além disso, medidas de proteção contra gotículas devem ser adotadas como o uso de máscara cirúrgica ou caseira ao manusear e alimentar seu bebê, sendo realizada a substituição caso esteja úmida ou ao tossir, espirrar e a cada mamada, caso a mãe não possua máscara facial, utilizar um pano limpo cobrindo boca e nariz, higienização das mãos antes e após o contato com a criança, manter uma distância segura de dois metros do bebê nos momentos em que não estiver o manipulando e realizar a limpeza e desinfecção das superfícies próximas à mãe (GIULIANI C, et al., 2020; BRASIL, 2020)

Nas situações nas quais a mãe não tem condições de amamentar, recomenda-se que seja feita a extração do leite e esse deve ser oferecido in natura para o RN por meio de copinho ou colher. Antes da ordenha, deve ser realizada a higiene das mãos e utilizada a máscara cirúrgica. Caso sejam utilizados acessórios para ajudar na retirada do leite, toda a superfície deve ser desinfetada com álcool a $70 \%$ e os acessórios devem ser lavados e fervidos por 15 minutos, a contar do momento da ebulição da água (MOCELIN HJS, et al., 2020).

Uma observação importante destacada pela literatura é que, se a extração do leite for feita por bomba de leite manual ou elétrica, a mãe deve lavar as mãos antes de tocar em qualquer bomba ou frasco e seguir as recomendações para limpeza adequada do aparelho (GIULIANI C, et al., 2020). Também é recomendado que - leite materno seja ofertado por uma pessoa saudável, que deve receber treinamento de um profissional qualificado antes de iniciar os procedimentos (MARTINS- FILHO PR, et al., 2020).

$\mathrm{Na}$ impossibilidade da ordenha do leite devido às condições de saúde materna, ou pelo uso de medicações incompatíveis com a amamentação, há a opção de recorrer aos Bancos de Leite Humano (BLH). Espera-se que durante a pandemia devido ao isolamento social ocorra um menor número de doações, afetando assim o abastecimento das unidades de terapia intensiva neonatais (UTIN) e aumento a oferta de fórmula infantil (FERNÁNDEZ-CARRASCO FJ, 2020; CARDOSO PC, et al., 2021).

Algumas medidas foram tomadas para que a doação de leite seja mantida de forma segura neste período, dentre elas é a contraindicação de doação por mães que apresentem sintomas clássicos de síndrome gripas, infecção respiratória ou diagnóstico de COVID-19. Mulheres que relatem ter tido contato próximo com indivíduos com infecção suspeita ou confirmada também não poderão realizar a doação de leite materno (CARDOSO PC, et al., 2021). 
Em contraposição à continuidade da amamentação no caso de COVID-19, entidades chinesas acreditam que todos os bebês suspeitos ou filhos de mulheres confirmadas por COVID-19 devem ser internados em UTIN, sendo todos eles rastreados para COVID-19 por meio da coleta de história clínica e não clínica de seus pais. Também devem realizar o teste PCR-RT nas primeiras 24 horas de vida através de swabs separados da nasofaringe, orofaringe e reto. Um segundo teste 24 horas depois parece valioso para confirmação da doença (SIGHALDEH SS e KALAN ME, 2020).

Os RNs de alto risco devem ser isolados por pelo menos 14 dias em sala separada. Essas medidas são justificadas pelo sistema imunológico dos bebês que ainda está em desenvolvimento. Eles enfatizam que, durante esse período de isolamento, as mães não devem alimentar seus filhos com leite materno até sua recuperação. Tais orientações são justificadas por 2 casos de infecção neonatal por coronavírus, suspeita-se que ocorreram pelo contato pós-natal com a mãe infectada (SIGHALDEH SS e KALAN ME, 2020; MOCELIN HJS, et al., 2020).

Embora as orientações tenham sido sugeridas, apenas para mulheres com testes positivos para SARSCOV-2, este aviso pode contribuir para redução do aleitamento materno, o que pode ocasionar vários efeitos negativos para mãe e seu filho. Portanto, essa conduta de separar o binômio deve ser limitada a situações extremas e apoiada por boas evidências científicas (MARTINS-FILHO PR, et al., 2020; LUBBE W, et al., 2020).

\section{CONSIDERAÇÕES FINAIS}

Até o presente momento, não há evidências sobre a presença do SARS-COV-2 no leite materno de puérperas com COVID-19. Os dados fornecidos na literatura são limitados e a amamentação nessas mulheres permanece uma questão controversa. A grande maioria das evidências encontradas recomendam que a amamentação deve ser continuada, porém as medidas de precauções devem ser mantidas e reforçadas criteriosamente. Além da manutenção das medidas preventivas gerais, os profissionais da saúde devem oferecer apoio e estímulo à amamentação. Durante a busca nas bases de dados foi observado um número reduzido de publicações, isso é justificável pela contemporaneidade da temática o que gera uma lacuna para o conhecimento mais aprofundado sobre o COVID-19 e amamentação.

\section{REFERÊNCIAS}

1. BRASIL. Ministério da Saúde. Trata-se de avaliação de medida para o enfrentamento da emergência em saúde pública no contexto da infecção da COVID-19 causada pelo novo coronavírus (SARS-CoV-2), sobre questionamento da Rede de Atenção à Saúde (RAS) respectivo à preservação da amamentação em situação de risco iminente de transmissão do respectivo vírus, em situações que a mãe apresente sintomatologia compatível com síndrome gripal. 2020. Disponível em: https://portaldeboaspraticas.iff.fiocruz.br /biblioteca/nota-tecnica-no-9-2020-cosmu-cgcivi-dapes-sapsms/. Acessado em: 08 de março de 2020.

2. BRASIL. Ministério da Saúde. Atenção ao Recém-Nascido e COVID-19.2020. Disponível em: https://egestorab.saude.gov.br/image/?file=20200805_N_NotaTecnicaCovidCocam15_8045946382474299533.pdf. Acessado em: 02 de abril de 2020.

3. CARDOSO PC, et al. A saúde materno-infantil no contexto da pandemia de COVID-19: evidências, recomendações e desafios. Rev. Bras. Saude Mater. Infant., 2021; 21 (supl. 1): 213-220.

4. CENTENO-TABLANTE E, et al. Transmission of SARS-CoV-2 through breast milk and breastfeeding: a living systematic review. Ann. N.Y. Acad. Sci, 2020; 1484: 32-54.

5. $\mathrm{CHEN} \mathrm{H}$, et al. Clinical characteristics and intrauterine vertical transmission potential of COVID-19 infection in nine pregnant women: a retrospective review of medical records. Lancet, 2020; 395(10226): 809-15.

6. COJOCARU L, et al. An initiative to evaluate the safety of maternal bonding in patients with SARS-CoV-2 infection. J Matern Fetal Neonatal Med., 2020; 30: 1-7.

7. FERNÁNDEZ-CARRASCO FJ, et al. Infección por coronavirus Covid-19 y lactancia materna: una revisión exploratoria. Rev Esp Salud Pública, 2020; 94: 27.

8. GIULIANI C, et al. Breastfeeding during the COVID-19 pandemic: suggestions on behalf of woman study group of AMD. Diabetes Res Clin Pract., 2020; 165: 108239.

9. GOES FGB, et al. BOAS PRÁTICAS NO CUIDADO AO RECÉM-NASCIDO EM TEMPOS DE COVID-19: REVISÃO INTEGRATIVA. Texto contexto - enferm., 2020; 29: e20200242.

10. GUIMARÃES DC, et al. Conhecimento da puérpera sobre amamentação na Atenção Básica. Revista Eletrônica Acervo Saúde. 2018; 18: e107. 
11. LACKEY KA, et al. SARS-CoV-2 and human milk: What is the evidence? Maternal \& Child Nutrition, 2020; 16(4): e13032.

12. LIMA ACMACC, et al. Consultoria em amamentação durante a pandemia COVID-19: relato de experiência. Esc. Anna Nery Rio de Janeiro, 2020; 24: e20200350.

13. LIU X, et al. Prenatal anxiety and obstetric decisions among pregnant women in Wuhan and Chongqing during the COVID-19 outbreak: a cross-sectional study. BJOG, 2020; 127(10): 1229-1240.

14. LUBBE W, et al. Breastfeeding during the COVID-19 pandemic - a literature review for clinical practice. International Breastfeeding Journal, 2020; 15 (1): 82.

15. MARCHIORI GRS, et al. Ações da enfermagem nos bancos de leite humano em tempos de COVID-19. Rev. Bras. Enferm., 2020; 73(supl. 2): e20200381.

16. MARTINS ABM, et al. Aleitamento materno e seu conhecimento por alunos de enfermagem. Nursing (São Paulo), 2020; 23(267): 4430-4435.

17. MARTINS-FILHO PR, et al. To breastfeed or not to breastfeed? Lack of evidence on the presence of SARSCoV- 2 in breastmilk of pregnant women with COVID-19. Rev Panam Salud Publica, 2020; 44: e59.

18. MASCARENHAS VHA, et al. Recomendações assistenciais à parturiente, puérpera e recém-nascido durante a pandemia de COVID-19: revisão de escopo. Rev. Latino-Am. Enfermagem, 2020; 28: e3359.

19. MELNYK BM, FINEOUT-OVERHOLT E. Evidence-based practice in nursing \& healthcare. A guide to best practice. China: Wolters Kluwer/Lippincott Williams \& Wilkins; 2011.

20. MENDES KDS, et al. Revisão integrativa: método de pesquisa para a incorporação de evidências na saúde e na enfermagem. Texto contexto - enferm., 2008; 17(4): 758-764.

21. MIRANDA PIG, et al. Ações realizadas na atenção primária à saúde às pessoas com transtorno mental: revisão integrativa. Rev Rene, 2021; 22: e60496.

22. MOCELIN HJS, et al. Panorama sobre as recomendações para amamentação em tempos de COVID-19. J. Hum. Growth Dev., 2020; 30(3): 335-343.

23. NG QJ, et al. Perception and Feelings of Antenatal Women during COVID-19 Pandemic: A Cross-Sectional Survey. Ann Acad Med Singap., 2020; 49(8): 543-552.

24. OMS. Organização Mundial de Saúde. Aleitamento materno e a doença causada pelo novo coronavírus (COVID-19) (23 de junho de 2020). 2020. Disponível em: https://www.paho.org/pt/documents/breastfeeding-and-covid-19scientific-brief-23-june-2020. Acessado em: 08 de março de 2021.

25. PERRINE CG, et al. Implementation of Hospital Practices Supportive of Breastfeeding in the Context of COVID-19 United States. MMWR Morb Mortal Wkly Rep., 2020; 69(47): 1767-1770.

26. SALVATORE CM, et al. Neonatal management and outcomes during the COVID-19 pandemic: an observation cohort study. The Lancet Child \& Adolescent Health, 2020; 4(10): 721-727.

27. SIGHALDEH SS, KALAN ME. Care of newborns born to mothers with COVID-19 infection; a review of existing evidence. J Matern Fetal Neonatal Med, 2020; 23: 1-13.

28. TACLA MT, et al. Reflexões sobre o aleitamento materno em tempos de pandemia por COVID-19 Rev Soc Bras Enferm Ped., 2020; 20(Especial COVID-19): 60-76.

29. YASSA M, et al. Near-term pregnant women's attitude toward, concern about and knowledge of the COVID-19 pandemic. The Journal of Maternal-Fetal \& Neonatal Medicine, 2020; 33(22): 3827-3834.

30. ZHU C, et al. Breastfeeding Risk from Detectable Severe Acute Respiratory Syndrome Coronavirus 2 in Breastmilk. J Infect., 2020; 81(3): 452-482. 